\title{
Estrategias innovadoras para favorecer el desarrollo de competencias. Aplicación en la enseñanza de ingeniería de las reacciones químicas.
}

Dra. María Fernanda Zalazar ${ }^{*_{a}}$; Ing. Qco. José Pablo Arquier ${ }^{b}$

RESUMEN: Se describe en espacio y tiempo una propuesta de enseñanzaaprendizaje innovadora implementada en alumnos de cuarto año cursando la asignatura de Ingeniería de las Reacciones de la carrera de Ingeniería Química de la Facultad Regional Resistencia de la Universidad Tecnológica Nacional. La situación problemática aborda la complejidad de diversos aspectos que intervienen en ella durante 8 períodos académicos en los cuales ha sido desarrollada. La metodología fue implementada con el fin de desarrollar competencias profesionales articulando contenidos de la asignatura con la aplicación en el campo profesional a través de una visita a industria química de la región.

Palabras clave: visitas de campo, interacción universidad-industria, competencias profesionales

\section{INTRODUCCIÓN}

La propuesta de innovación se enmarca en la asignatura Ingeniería de las Reacciones correspondiente al cuarto nivel de la carrera de Ingeniería Química de la Facultad Regional Resistencia de la Universidad Tecnológica Nacional. Los objetivos de la asignatura, consisten en que los alumnos, además de aprender los contenidos específicos de la misma, tengan una visión general del contexto y de la aplicación de los temas de la asignatura en la disciplina, que sean capaces de integrar los conocimientos aprendidos (tanto de esta asignatura, como de otras del nivel) en relación a un proceso real, que desarrollen competencias de relaciones interpersonales y que sean capaces de comunicarse utilizando terminología específica de la asignatura.

La planificación de tareas se piensa tomando como base a la experiencia docente

\footnotetext{
a Ingeniería Química, Facultad Regional Rcia., Universidad Tecnológica Nacional. French 414 - 3500 Rcia, Chaco 'Indunor S.A.I.C.A. San Martín s/n - 3514 La Escondida - Argentina

*E-mail: mfzalazar@conicet.gov.ar
} 
de la cátedra, a través de la cual se detecta la necesidad de desarrollar en los alumnos algunas habilidades que están relacionadas por ejemplo; con la falta de apreciación de la realidad profesional, capacidad para análisis crítico, toma de decisiones, comunicación oral utilizando terminología ingenieril, integración de conocimientos, entre otras. En otras palabras, se les dificulta imaginar situaciones reales que corroboren las teorías o problemas prácticos que el docente explica en clase, no ven un problema real como un todo sino como partes aisladas, o no tienen noción de las dimensiones reales de equipos que ven en una filmina o en una imagen en un libro de la especialidad. Por ejemplo, asocian "salida de vapor de una chimenea" con salida de "polvos contaminantes".

En relación con esto último, se deduce que, salvo algunas excepciones, el contacto que tiene el alumno que cursa este nivel con la industria es escaso y por ende tiene pocos conocimientos de las industrias de la zona y los productos que se producen en ellas, mientras que en algunos casos, se identifica en alumnos que han visitado fábricas previamente, la falta de criterios formados.

En este sentido, Conteras Domingo (1990:4), mencionado en (Lucarelli, 2009), sostiene que "se puede pensar la enseñanza como "la elaboración de estrategias para entender y acortar las distancias entre las condiciones de realidad y las aspiraciones educativas", dado que se hace necesario conocer la realidad sobre lo que se actúa" $(\mathrm{p}: 36)$
A raíz de ello, se comenzó a diseñar a partir del año 2009, una experiencia educativa innovadora de visita a una industria química de la región, donde se trabajó en la propuesta junto a el responsable de la industria. A partir de las misma se planificó la visita sectores de interés de la industria y el desarrollo de un seminario con temáticas específicas comunes de la asignatura y la industria en cuestión.

La propuesta inicial se implementó en la cátedra a partir del año 2010, como motivación y vehículo para el desarrollo de competencias de los alumnos, para ayudarlos a mejorar sus conocimientos y ampliar sus horizontes, proporcionándoles así, una visión in situ de las aplicaciones vistas en la asignatura. Asimismo, la propuesta constituye desde la perspectiva de los docentes una opción para mejorar la enseñanza de la asignatura, incorporar requerimientos para acreditación de carreras de ingeniería, generar vínculos entre la universidad y la industria, y contribuir a mejorar la calidad educativa (Zalazar y Arquier, 2012).

En el periodo 2010-2018 desde su implementación hasta la fecha, la experiencia se ha realizado de manera continua, y año tras año se han ido incluyendo mejoras continuas en el desarrollo de la misma. En este trabajo se describe la propuesta, las innovaciones que se han ido realizando a lo largo de los años y el impacto de la experiencia en la formación integral de los alumnos. Asimismo, se aprecia el nivel de compromiso de la industria involucrada, para que dicha actividad se pueda realizar de manera continua en la asignatura. 


\section{DESARROLLO}

Contextualización: La asignatura Ingeniería de las Reacciones Químicas, es una asignatura específica de la carrera de Ingeniería Química, de dictado cuatrimestral, con una carga horaria de $10 \mathrm{hs}$ semanales. E1 promedio anual de alumnos es de 30 a 40 alumnos. Plantea contestar los interrogantes que el ingeniero químico enfrenta en situaciones comunes en su área de trabajo, ¿Cuál es la información necesaria para atacar un problema?, ¿Cuál es la mejor manera de obtenerla?, ¿Cómo seleccionar el diseño apropiado entre las diferentes opciones? Para su desarrollo, se cuenta con los recursos mínimos necesarios. Si bien la facultad posee una planta piloto, no dispone de equipos específicos para llevar a cabo prácticos relativos a la asignatura (ejemplo reactores de diverso tipo). Es por ello que la visita a una industria química en particular se plantea como un espacio físico que puede acercar a los alumnos a una visión de la realidad profesional.

Fundamento pedagógico: Elisa Lucarelli, entiende que la innovación es aque1la práctica protagónica de enseñanza o de programación de la enseñanza, en la que, a partir de la búsqueda de la solución de un problema relativo a las formas de operar con uno o varios componentes didácticos, se produce una ruptura en las prácticas habituales que se dan en el aula de clase, afectando el conjunto de relaciones de la situación didáctica (Lucarelli et al. s/f). En síntesis, la innovación es comprendida como:
- Ruptura con un modelo estereotipado de la enseñanza y del aprendizaje,

- Selección creadora, organización y utilización de recursos en una forma nueva y original que permita alcanzar objetivos definidos,

- Ruptura del statu quo: conjunto de prácticas que abarcan: 1o didáctico curricular, psicosocial y administrativo de la tarea pedagógica,

- Con carácter histórico, situacional, en tanto se tiene en cuenta la importancia de la génesis y el desarrollo para la comprensión, modificación o alteración de una situación dada, con propósitos de mejorarla.

La experiencia de enseñanza-aprendizaje innovadora se enmarca en el desarro1lo de un trabajo de campo, específicamente una visita a una industria de procesos químicos, la que se complementa con la incorporación de diversas herramientas TIC's para favorecer la comprensión e integración de contenidos, así como estimular procesos cognitivos de síntesis y de gestión de contenidos; que se orienta al desarrollo de competencias profesionales y personales en los alumnos.

La propuesta surge inicialmente, como respuesta a uno de los requerimientos de la CONEAU, para cumplir con estándares de acreditación de carreras de ingeniería (Resol ME 1232/01), que establecen que es necesario incluir en la currícula de la carrera, un conjunto de experiencias apropiadas en laboratorios, talleres o trabajos de campo que sirvan para combinar elementos de teoría con la práctica y parale- 
lamente propiciar que el alumno adquiera, además de los conocimientos específicos de la asignatura, habilidades y destrezas transversales que los capaciten para el futuro profesional; evoluciona luego a través de los años con la incorporación de actividades que le otorgan al alumno un rol activo.

La innovación a través de esta actividad consiste entonces en transformar la docencia universitaria tradicional centrada en una enseñanza magistral y basada en los contenidos de las disciplinas, trasladando su eje desde la enseñanza hacia el aprendizaje participativo. Así, el énfasis está puesto en el trabajo autónomo de los estudiantes y orientado a la organización de la enseñanza por competencias, lo que exige una revisión de la forma de planificar y desarrollar la docencia. Por lo tanto, el aprendizaje basado en competencias, es el resultado de la integración de experiencias de aprendizaje, donde las habilidades, destrezas y conocimientos interactúan para formar un nuevo conocimiento, de forma tal que cumplen con la tarea para el cual fue diseñado.

De acuerdo con Zoppi (1999), las innovaciones se pueden encuadrar desde la "actualización de enfoques y ajuste de ejes temáticos y contenidos". En este rubro, el docente innova modificando las prácticas como medio para la transformación de los contenidos, y en relación a ello se advierte un posicionamiento distinto al promover criticidad, uso flexible, etc; que requiere ineludiblemente la transformación de las prácticas docentes tanto en la situación de enseñanza como en la de evaluación. En este encuadre se ajusta parte de la primer parte de la experiencia propuesta, que involucra integrar contenidos y desarrollar competencias a partir del trabajo de campo.

En esto radica la diferencia de las visitas a la planta industrial en los primeros años que se desarrolló la experiencia (2010-2011), en las cuales no se planteaban actividades de tipo participativo-movilizadoras de los aprendizajes. Los alumnos se limitaban a observar y solo algunos de ellos se animaban a preguntar, quizás por temor a hacer preguntas fuera de contexto. Esto planteó la necesidad de que los alumnos, como actividad previa a la visita, se familiaricen con la industria y los procesos que en ella se realizan, de modo de aprovechar al máximo la experiencia.

Es así que, en el año 2012, se incorpora al trabajo de campo una WebQuest (Area Moreira, s/f), que consiste en actividades de aprendizaje que se llevan a cabo utilizando recursos de Internet preseleccionados por el docente, de manera que el estudiante se enfoque en el uso de los recursos y no en su búsqueda. Como se sabe, internet posibilita la obtención de información que está disponible en muchos sitios. Sin embargo, el principal problema es la calidad de esta información, en especial cuando es utilizada por los alumnos para fines académicos sin criterios formados de selección. Así, esta herramienta se implementa para guiar al alumno en la búsqueda de información específica relativa a la empresa en este caso, que no forma parte de la 
bibliografía establecida en la carrera ( $\mathrm{Za}$ lazar y Arquier, 2013). Constituye también, un modo de promover experiencias innovadoras en los procesos de enseñanzaaprendizaje apoyándose en el uso de las TIC's. Además, a partir de ellas se les proporciona a los alumnos una tarea bien definida, así como los recursos y las consignas que les permitan realizarlas (Dodge, 1995; Barba, 2002).

Esta innovación, además de la categoría de "aplicación de nuevos medios para la enseñanza y el aprendizaje" propuesto por Zoppi (1999,p:186), se encuadra y trae aparejado consigo también entrar en la categoría de "adopción de una concepción de aprendizaje activo" (Zoppi, 1999, p:179) donde se busca que el alumno tome un rol más activo, así como un replanteo en el rol docente, se articulan diversas fuentes de información y vías para el conocimiento, $y$ las tareas se focalizan en determinados rasgos de los perfiles profesionales. Estos últimos acercando al estudiante al campo de sus futuras incumbencias, a través de esta experiencia a un lugar de trabajo real.

Otro cambio, en relación a las primeras visitas o trabajo de campo y las de los últimos años, se relaciona con la evaluación que si bien inicialmente se incluyeron instancias evaluativas, éstas estaban destinadas a solo algunos aspectos de la innovación, y más desde la perspectiva de evaluar la actividad desde el punto de vista docente y no tanto de los logros alcanzados por los alumnos. Como resultado de la reflexión docente en y sobre la acción, se contempló la necesidad de implementar algún tipo de evaluación de la actividad, que permita cubrir ese déficit. Es así que a modo de cierre de la actividad y a través de la WebQuest, se les plantea a los alumnos, realizar una producción grupal escrita que luego será evaluada, incluyendo también una reflexión individual.

Para Zoppi (1999:87) "Evaluar el aprendizaje de los alumnos le permite al docente comprender qué tipos de procesos realiza el alumno. Qué es lo que han comprendido y qué es lo que no ban asimilado. Pero además, constituye una fuente inagotable de interrogantes para los alumnos y para los mismos docentes. Les permite a éstos, repensar la innovación, rever sus estrategias y encontrar los incentivos necesarios para continuar en el proceso creativo de la innovación."

De este modo, la experiencia formulada a partir del 2009 e implementada desde el año 2010, ha ido adquiriendo mejoras continuas teniendo en cuenta los comentarios de alumnos, las ideas originadas desde el docente y desde el responsable de la industria, así como también en base a la experiencia adquirida.

Objetivos: Los objetivos generales planteados en la propuesta están enfocados en el desarrollo de competencias relacionadas con la formación personal y profesional del alumno, y al mismo tiempo introducirlo en los procesos característicos de la profesión.

Los objetivos particulares planteados desde la perspectiva del alumno implican:

- Identificar las distintas unidades de 
proceso (por ej. tipo de reactores: homogéneo, heterogéneo, discontinuo, semicontinuo o continuo, mezcla completa ó flujo pistón)

- Describir las características básicas de los procesos que se desarrollan en sectores industriales regionales de la Ingeniería Química.

- Observar las funciones y tareas de los ingenieros químicos en su puesto de trabajo

- Interaccionar con profesionales del área de la industria, recopilar información inherente al proceso, a las variables de control y a las condiciones de operación.

- Utilizar la terminología específica adquirida en la cátedra para expresar apropiadamente las observaciones realizadas sobre el ámbito industrial.

- Visualizar las dimensiones de los equipos, apreciar su funcionamiento y percibir como se lleva a cabo una reacción química en el ámbito industrial

- Adquirir una visión a escala real de los equipos e instalaciones auxiliares que intervienen en las operaciones.

Por otra parte, los objetivos de la incorporación de TIC's están enfocados en:

- Buscar, analizar e integrar información utilizando Internet como principal fuente de información.

- Construir, sintetizar y elaborar un informe trabajando de manera colaborativa, a partir de la información recabada.

- Desarrollar competencias relacionadas con su formación personal y profesional: captación de información no estructurada, trabajo en equipo, creatividad, habilidades comunicativas, flexibilidad, planificación, contextualización e integración de conoci- mientos.

- Valorar las ventajas que aporta Internet para favorecer la investigación respecto a un tema específico, y no disponible en la bibliografía convencional.

- Utilizar un foro como herramienta de intercambio de información y debate.

Plan de Actividades: Para organizar una actividad como formadores debemos preguntarnos, qué tipo de competencias deben adquirirse, por medio de que métodos y en que ámbitos de la práctica. De esta manera "el alumno tiene que ver por si mismo y a su propia manera las relaciones entre los medios y los métodos empleados y los resultados conseguidos. Nadie más puede verlo por él, y no puede verlo simplemente por alguien que se lo diga”. (Schön, 1992). Es así que la propuesta actual involucra las plasmadas en la Figura 1. Las actividades de investigación planteadas en la primer semana, están elaboradas en una WebQuest disponible a través del aula virtual de la asignatura que se encuentra alojada en el campus virtual de la facultad. Para la semana 2, la visita se encuentra organizada siguiendo la Figura 2.

A través de ésta experiencia se promueve en los alumnos la capacidad de relacionar los parámetros de operación, visualizan el rol que puede tomar un ingeniero químico en la industria, integran conocimientos de la asignatura, en conjunto con otras asignaturas del nivel como operaciones unitarias, tecnología del calor, etc, y pueden ver además de todas las instalaciones anexas a los reactores (válvulas, cañerías, equipos auxiliares, etc.). Foto 1 


\begin{tabular}{|c|c|c|}
\hline Semana 1 & Semana 2 & Semanas 3-4 \\
\hline $\begin{array}{l}\text { - Docentes } \\
\text { - Definición objetivos } \\
\text { - Exposición actividades } \\
\text { utilizando prezi } \\
\text { - Introducción plantas a } \\
\text { visitar } \\
\text { - Presentación actividades } \\
\text { previas a realizar } \\
\text { - Presentación WebQuest } \\
\text { - Coordinación con } \\
\text { industria, facultad y } \\
\text { gestion de recursos } \\
\text { - Cocrdinación y } \\
\text { organización con } \\
\text { alumnnos } \\
\text { - Alumnos } \\
\text { - Investigación guiada por } \\
\text { webquest }\end{array}$ & $\begin{array}{l}\text { - Docentes } \\
\text { - Facilita comprensión } \\
\text { - Dinamizar el grupo } \\
\text { - Alumnos } \\
\text { - Visita a Industria } \\
\text { - Observación } \\
\text { - Consultas al ing Qco y } \\
\text { personal de la empresa } \\
\text { - Recopilación de } \\
\text { información } \\
\text { - proceso } \\
\text { - variables de control } \\
\text { - condiciones de } \\
\text { operación } \\
\text { - Analizan e integran } \\
\text { conocimientos. } \\
\text { - Responden una encuesta }\end{array}$ & $\begin{array}{l}\text { - Docentes } \\
\text { - Evaluación de los } \\
\text { informes } \\
\text { - Evaluación de la } \\
\text { experiencia } \\
\text { - Moderador del foro } \\
\text { - Alumnos } \\
\text { - Análisis de la información } \\
\text { y observaciones } \\
\text { recopiladas en la } \\
\text { experiencia } \\
\text { - Participación en el foro } \\
\text { - Proceso de sintesis y } \\
\text { gestión del contenido y } \\
\text { elaboración del informe }\end{array}$ \\
\hline
\end{tabular}

Figura 1: Plan de actividades

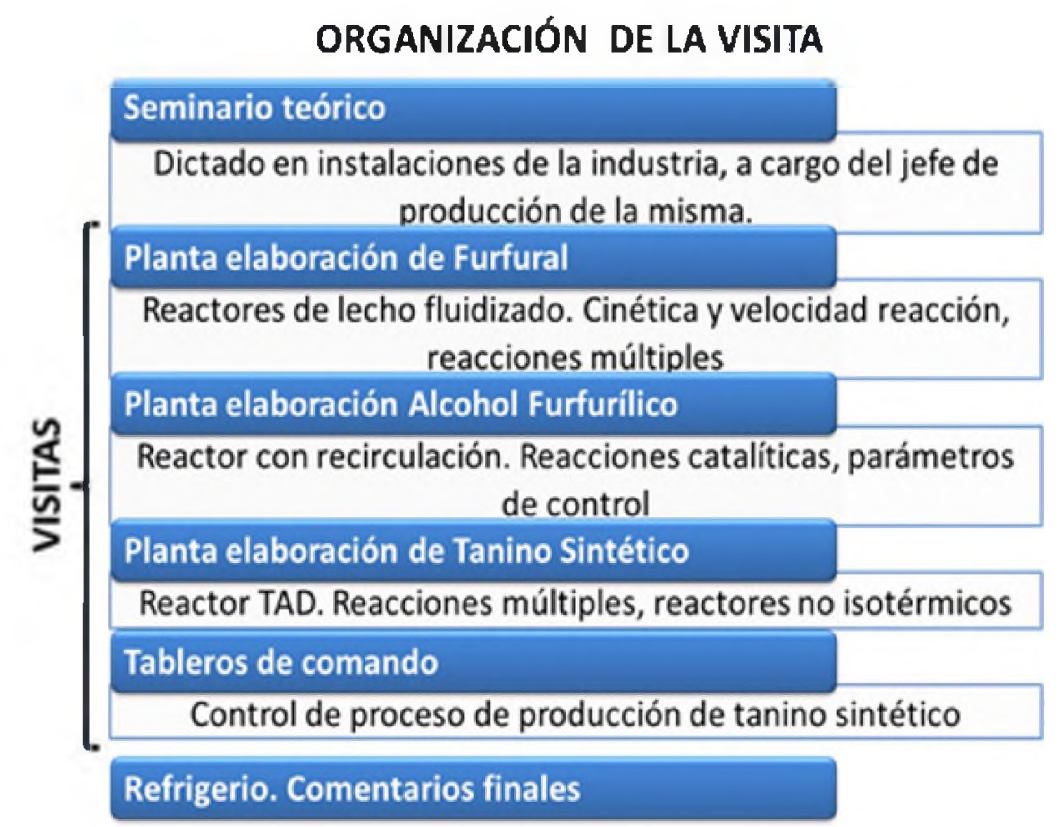

Figura 2: Organización de las actividades desarrolladas durante la visita 


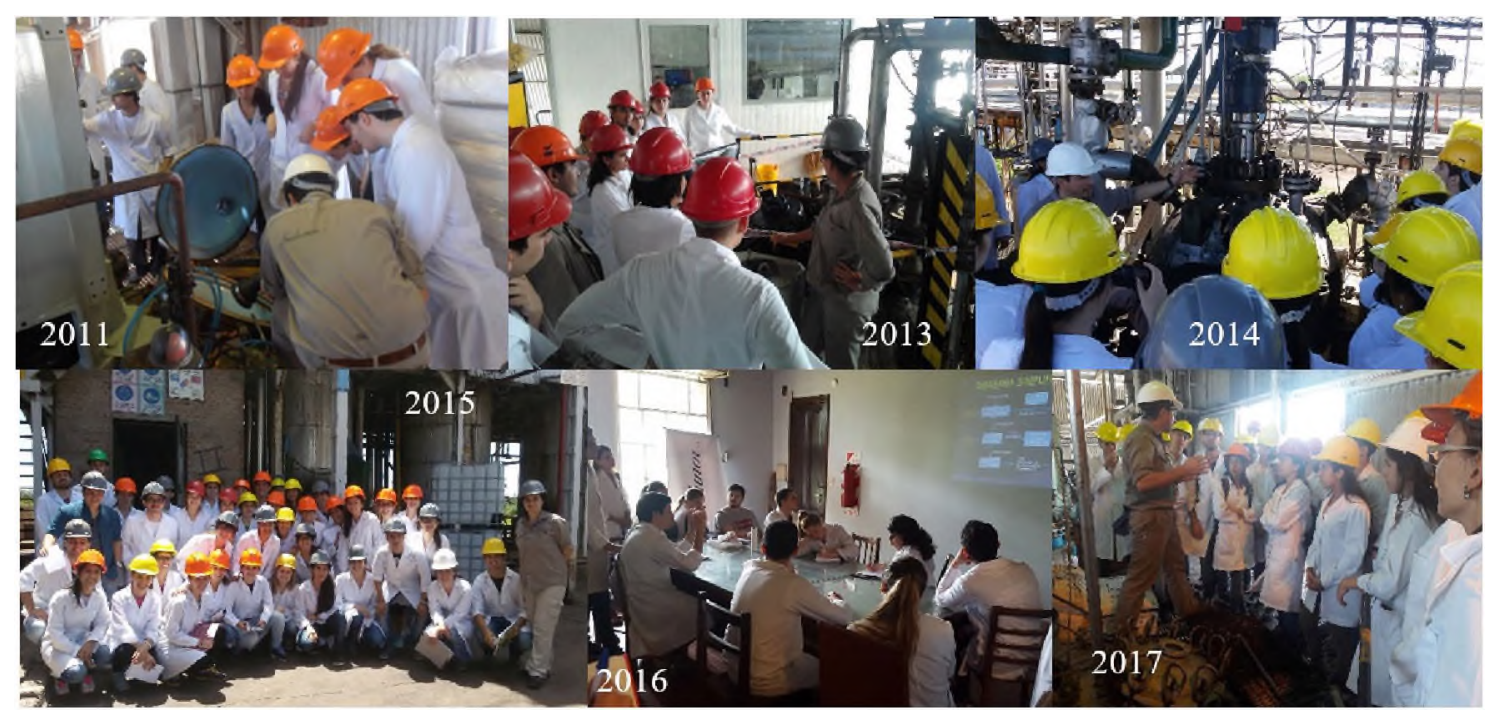

Foto 1: Alumnos de diferentes cohortes participando de la visita a industria

En las semanas 3 y 4, como complemento adicional a las actividades se habilita un "foro de intercambio de información y debate" en el aula virtual de la asignatura, de manera que los alumnos intercambien información, colaboren con sus compañeros, discutan grupalmente, compartan ideas (y respeten opiniones), y estimulen así su capacidad crítica. Con toda la información recabada, deben elaborar un informe, cuyo formato y contenidos se detalla en la WebQuest previamente comentada. Asimismo, a través del aula virtual realizan una encuesta para comunicar su opinión sobre como contribuye esta actividad a afianzar los conceptos aprendidos en clase, y sugieren modificaciones o como mejorar la experiencia.

La propuesta fue evolucionando con los años, actualmente los objetivos de la misma son más amplios que aquellos plan- teados inicialmente. Para la visita del 2017 (luego de llevar 7 años realizando esta actividad), los resultados tanto de la visita, como de escuchar a los alumnos durante la experiencia, visualizar como interaccionan con los profesionales de la industria, como identifican conceptos trabajados en la asignatura, y evaluar al final el producto integrado y elaborado que entregan en sus informes, permite apreciar que los alumnos alcanzaron a apropiarse de los saberes para los cuales estaba planteada la actividad. Analizándolo desde la perspectiva del alumno, se considera por sus reflexiones que la experiencia para ellos es gratificante y superadora generando un impacto en su formación integral. Esto es un indicador de que los alumnos logran lo que Díaz $\mathrm{Ba}^{-}$ rriga identifica como aprendizaje signiftcativo, para este autor aprender significa construir sentido respecto de un objeto. A 
continuación, se transcriben algunas reflexiones de los alumnos:

".. No habia tenido la oportunidad de visitar otras industrias anteriormente. Esto me permitió tomar real dimensión del alcance que un Ingeniero Quimico de fábrica tiene que manejar y conocer. Fue importante resaltar que la formación que se adquiere en la facultad permite tener noción técnica sobre los procesos involucrados, pero que una planta tiene muchos otros factores que se deben considerar: como lo social, económico y otras competencias ajenas al cálculo, que el ingeniero deberá aprender a lidiar. La visita permite visualizar y comprender con detalles todos los equipos que en los croquis de los libros dificultan el entendimiento..." (Alumno cohorte 2016)

"Personalmente me paso que si bien tenia una idea, $y$ al ver los equipos iba tratando de relacionar con lo que veniamos aprendiendo en la asignatura, bice el trabajo inverso, es decir, luego de la visita, estudiando para el segundo parcial, me iba acordando de lo que habiamos visto y eso me ayudaba a entender o a imaginarme como ocurren los procesos vistos. Otra cosa que logré con la visita es dimensionar como son realmente los equipos, su tamaño, materiales, las condiciones de operación." (Alumno cohorte 2017)

Analizando la propuesta como innovación, algunas de las innovaciones se incorporaron a través del uso de diversas estrategias de enseñanza de articulación teoría-práctica y que colocan al alumno en un rol participativo. De acuerdo a Sanjurjo, la articulación teoría-práctica no sólo persigue la comprensión y la interpretación, sino también la toma de conciencia de las condiciones reales y de los contextos que posibilitarán la acción para el cambio (Sanjurjo, y otros, 2009). Desde esta perspectiva, se busca esta articulación a través la integración de contenidos de la asignatura y su aplicación en el campo profesional actual.

\section{CONCLUSION}

A nivel de la propuesta metodológica se observan las características que asume una propuesta de innovación pedagógica, como se fue gestando y desarrollando, como a lo largo de los años se buscó la articulación teoría-práctica y como dicha experiencia ha ido adquiriendo mejoras continuas, en un proceso que se va retroalimentando año a año. Desde su implementación hasta la fecha, en la cual se ha realizado de manera continua en estos últimos 8 años, se han ido incluyendo mejoras continuas en la experiencia, y se han obtenido muy buenos comentarios de la misma tanto de los alumnos como de la industria involucrada, la cual muestra un nivel de compromiso para que dicha actividad se pueda realizar de manera continua en la asignatura. Desde la perspectiva docente, se han cumplido los objetivos iniciales propuestos.

Sin embargo, resta mucho por hacer, para continuar con esta propuesta inno- 
vadora planteamos la necesidad de seguir innovando, se podría considerar la participación de los alumnos en el diseño de actividades, en la gestión de los recursos para el traslado, entre otras decisiones.

\section{AGRADECIMIENTOS:}

INDUNOR S.A.I.C.A. y Silvateam Group por permitir desarrollar la visita a su planta química en la Escondida y por la buena predisposición del personal para llevar a cabo este tipo de actividad. Secretaría académica de la FRRe-UTN.

\section{BIBLIOGRAFÍA}

Area Moreira, M. WEBQUEST. Una estrategia de aprendizaje por descubrimiento basada en el uso de Internet. Laboratorio de Educación y Nuevas Tecnologías de la Universidad de la Laguna. Extraído el 19 de agosto de 2014 desde: http://manarea.webs. ull.es/materiales/webquest/webquest.pdf

Barba, C. (2002). La investigación en Internet con las WebQuest. Comunicación y Pedagogía, 185, 62 .

Díaz Barriga, F. (2003) Cognición situada y estrategias para el aprendizaje significativo. Revista electrónica de Investigación educativa, 5, 2.

Dodge, B. (1995). WebQuest: A technique for Internet-based learning. Distance Educator, $1,10$.

Lucarelli, E. y otros. (s/f) La Innovación Pedagógica en el Aula Universitaria. Estudios de Casos en la UNS. Extraído el 19 de agosto de 2014 desde: http://rapes.unsl.edu.ar/ Congresos_realizados/Congresos/IV\%20
Encuentro\%20-\%20Oct-2004/eje3/16.htm Lucarelli, E. (2009) Teoría y práctica en la Universidad. La innovación en las Aulas. Buenos Aires: Miño y Dávila.

Resolución 1232/01 Ministerio de Educación de la Nación. http://www.coneau.edu.ar/archivos/538.pdf

Sanjurjo, L.; Caporossi, A.; España, A. E.; Hernandez, A.M.; Alfonso, I. y Foresi, M.F. (2009). Los dispositivos para la formación en las prácticas profesionales. Rosario: Homo Sapiens Ediciones

Schön, D. (1992) La formación de profesionales reflexivos. Hacia un nuevo diseño de la enseñanza y el aprendizaje en las profesiones. Buenos Aires: Paidós.

Zalazar, M. F. y Arquier, J. P. (2012) Interacción universidad-industria a través de experiencia de visita a planta química. En actas de las II Jornadas de Investigación en Ingeniería del NEA y Países Limítrofes: hacia dónde van la ciencia y la tecnología en el MERCOSUR, Junio, Resistencia. Disponible en: http://www.frre.utn.edu.ar/IIJCyT/clean/ files/get/item $/ 2173$

Zalazar, M. F. y Arquier, J. P. (2013) Propuesta metodológica para el desarrollo de competencias profesionales en estudiantes de ingeniería química. En actas del VII Congreso Argentino de Ingeniería Química. Octubre, Buenos Aires. Disponible en: http:// www.aaiq.org.ar/SCongresos/docs/04_025/ papers/08a/08a_1485_731.pdf

Zoppi, A. M. y otros (1999) Procesos autónomos de innovación curricular: una aproximación a los sentidos que estructuran su producción. Disponible en: www.anamariazoppi.com.ar. 